\title{
Überfällige Einführung in die politischen Systeme Amerikas in einem Band
}

\author{
Stüwe, Klaus und Stefan Rinke (Hrsg.): Die politischen Systeme in Nord-und Lateinamerika. \\ Eine Einführung, VS Verlag für Sozialwissenschaften, Wiesbaden 2008, € 49,90.
}

Das vom Verlag als Einführung angekündigte Buch war längst überfällig, denn während zu den USA und Kanada eine Vielzahl von Studien vorliegen, fehlt eine neuere Publikation, die die politischen Systeme Lateinamerikas zum Gegenstand hat. Mittlerweile muss das verdienstvolle „Handbuch der Dritten Welt" von Dieter Nohlen und Franz Nuscheler mit seinen beiden Bänden zu Süd- beziehungsweise Mittelamerika und der Karibik aus dem Jahr 1995 als überholt gelten; eine Neuauflage ist offenbar nicht geplant. Auch das von Peter Waldmann herausgegebene „Politische Lexikon Lateinamerika“ datiert von 1992.

Von einem Politikwissenschaftler und einem Historiker ediert, sieht sich der Band sowohl historischer als auch politikwissenschaftlicher Komparatistik verpflichtet. Der Vergleich ist keineswegs einfach, denn die Bezeichnung Nordamerika bezieht sich zunächst auf einen geografischen Raum (zu dem nicht wenige mittlerweile auch Mexiko zählen), Lateinamerika hingegen wird eher als ein kulturell-politischer Begriff verwendet. In der 50-seitigen Einleitung konzentrieren sich die Herausgeber auf vergleichende Aspekte und versuchen charakteristische Merkmale in der Entwicklung des Doppelkontinentes herauszuarbeiten. Erwähnt werden etwa die Unterschiede während der Kolonialzeit: Die Siedlungskolonisation in Nordamerika förderte die Ausprägung einer Zivilgesellschaft, in Lateinamerika beschränkte sich die Kolonisation weitgehend auf die Küstenregionen und auf die Extraktion von Edelmetallen und Rohstoffen. Daraus und aus der sozialen Schichtung ergaben sich ungünstige Voraussetzungen für die Demokratie. Auf die spezifische Ausformung der Parteien und Parteiensysteme des Doppelkontinents wird ansatzweise eingegangen. In den lateinamerikanischen Staaten zeigt sich eine spezielle Mischung zwischen eigenständigen, europäischen und nordamerikanischen Elementen. Einzelne Organisationen basieren nach wie vor auf dem Prinzip des demokratischen Zentralismus sozialistischer Parteien Europas, zeichnen sich aber gleichzeitig durch einen starken Personalismus aus und führen zumindest in den Ländern mit Ölvorkommen einen aufwendigen Wahlkampf nach nordamerikanischen Mustern. Solche Aspekte werden angedeutet; ausführlicher gehen Stefan Rinke und Klaus Stüwe auf die Auswirkungen des Präsidentialismus und die Präsidentialismus-Parlamentarismus-Debatte in Lateinamerika ein. Nur gestreift wird die Rolle der sozialen Bewegungen und die Bedeutung des neuen Populismus.

Die Verfasser der Länderbeiträge sind Sozialwissenschaftler oder Historiker. Ein aus 15 Punkten bestehendes Gliederungsschema dient als Richtschnur. Am Anfang steht die geschichtliche Entwicklung seit der Unabhängigkeit, es folgen Abschnitte zum Staatsoberhaupt, den Parteien, Interessenverbänden und Kirchen, Massenmedien etc. Wenn ein Land von einem Historiker behandelt wurde, schlägt sich dies teilweise im Inhalt des Artikels nieder. Dies trifft beispielsweise für den gelungenen Beitrag über Argentinien zu, der allerdings etwas veraltet ist. Die Anzahl der im Literaturverzeichnis zitierten Titel fällt unterschiedlich aus: von neun für Costa Rica bis 59 für Kanada; die Literaturliste zu Mexiko ist mehr als doppelt so lang wie die zu den USA. Einige Aufsätze verwenden Tabellen und Grafiken oder ziehen im Abschnitt über die Politische Kultur den auf empirischem Material beruhenden Latinobarómetro heran, andere aber nicht. Hier wäre bei einer wahrscheinlich bald fälligen Neuausgabe eine gewisse Angleichung wünschenswert. Auch ist zu emp- 
fehlen, zu jedem Land einige Daten und Fakten in standardisierter Form zu präsentieren, zum Beispiel die Namen der gewählten Präsidenten und die Wahlergebnisse seit der Anfang der 1980er Jahre einsetzenden Re-Demokratisierung.

Auf ein abschließendes Kapitel haben die Herausgeber - sinnvollerweise - verzichtet. Dies würde der Vielfalt auf dem lateinamerikanischen Kontinent, der in den Jahren 2004 bis 2008 durch wirtschaftliche Dynamik gekennzeichnet war und eine sehr viel jüngere Bevölkerung aufweist als Europa, nicht gerecht. Es ist auch kein Nachteil, dass sich die Autoren auf die geschichtlichen und politischen Aspekte konzentrieren und auf knapp 600 Seiten gar nicht erst den Versuch machen, die sozialen und wirtschaftlichen Probleme von fast 900 Mio. Menschen zu erklären. Sowohl in der Einleitung als auch in den meisten Länderartikeln wird das Bekenntnis zur Demokratie als eine Gemeinsamkeit von Nordund Lateinamerika betrachtet. Ob in späteren Ausgaben diese optimistische Sichtweise Bestand haben wird, kann erst die zukünftige Entwicklung zeigen. Aufgrund der sowohl historisch als auch politikwissenschaftlich ausgerichteten Konzeption des Bandes dürfte es möglich sein, in einer Neuausgabe auch den aktuellen Trend hin zu plebiszitären und populistischen Formen der Demokratie gerade in Südamerika zu berücksichtigen.

Nikolaus Werz

\section{Informative Einführungen in die sechs politischen Systeme Ostasiens}

Heberer, Thomas und Claudia Derichs (Hrsg.): Einführung in die politischen Systeme Ostasiens. VR China, Hong Kong, Japan, Nordkorea, Südkorea, Taiwan, VS Verlag für Sozialwissenschaften, 2. aktualisierte und erweiterte Auflage, Wiesbaden 2008, 463 Seiten, € 34,90.

Die Länder Ostasiens sind zum neuen Gravitationszentrum der Weltwirtschaft geworden und werden deshalb oft als ein einheitlicher Block betrachtet. Es heißt, dass sie alle zum so genannten „konfuzianischen Kulturkreis“ gehören und auch die Geschichte teilen, dass sie im 19. Jahrhundert gezwungen waren, sich in eine vom Westen dominierte internationale Weltordnung einzufügen. Dabei wird leicht die Tatsache ignoriert, dass die „konfuzianische Kultur" als gemeinsame kulturelle Wurzel dieser Region umstritten ist. Zudem vergisst man, dass diese Staaten im 20. Jahrhundert nicht nur eine rasante wirtschaftliche Entwicklung, sondern auch den radikalen Wandel ihrer politischen Systeme, ja tief greifende Brüche in ihren politischen Verhältnissen erlebt haben: von der traditionellen Monarchie zur Demokratie beziehungsweise zum Sozialismus. Zugleich wurden sie, durchaus auch mit eigenem Zutun, in die Weltkriege und in den Kalten Krieg hineingezogen, der in Ostasien bis heute nicht beendet ist, wie in der Teilung der koreanischen Halbinsel zu sehen ist.

Nicht zuletzt diese komplexen historischen Hintergründe waren verantwortlich dafür, dass bisher nur selten der Versuch unternommen worden ist, die politischen Systeme der Länder Ostasiens in ihrem gesamten Umfang zu untersuchen. Mit diesem von Thomas Heberer und Claudia Derichs herausgegebenen Band hat man endlich auch im deutschsprachigen Raum ein respektables Buch, das sich für Einführungsseminare in die politischen Systeme Ostasiens eignet. Seine einzelnen Artikel beruhen auf langjährigen wissenschaftlichen Auseinandersetzungen der Autoren mit ihrem Untersuchungsgegenstand. Dass die 